\title{
Research
}

\section{Detection and Assessment of Ecosystem Regime Shifts from Fisher Information}

\author{
$\underline{\text { Arunprakash T. Karunanithi }}^{1}$, Heriberto Cabezas $^{1}$, B. Roy Frieden $^{2}$, and Christopher W. Pawlowski ${ }^{3}$
}

\begin{abstract}
Ecosystem regime shifts, which are long-term system reorganizations, have profound implications for sustainability. There is a great need for indicators of regime shifts, particularly methods that are applicable to data from real systems. We have developed a form of Fisher information that measures dynamic order in complex systems. Here we propose the use of Fisher information as a means of: (1) detecting dynamic regime shifts in ecosystems, and (2) assessing the quality of the shift in terms of intensity and pervasiveness. Intensity is reflected by the degree of change in dynamic order, as determined by Fisher information, and pervasiveness is a reflection of how many observable variables are affected by the change. We present a new robust methodology to calculate Fisher information from time series field data. We demonstrate the use of Fisher information to detect regime shifts on a model for a shallow lake. Next, we use Fisher information to analyze marine ecosystem response to physical changes using real time-series data of a coastal marine ecosystem, the North Pacific Ocean.
\end{abstract}

Key Words: ecosystems; Fisher information; marine ecosystem; regime shifts; resilience; sustainability.

\section{INTRODUCTION}

The study of regime shifts in complex dynamic systems has been quickly gaining importance because of the implications that regime shifts can have for modern society. Of particular importance to the United States Environmental Protection Agency (U.S. EPA) is the fact that sustainability is about finding and maintaining a regime suitable to the social and economic development of humans without major consequences to the environment, e. g., land, atmosphere, and ocean, and the ecological services on which people depend. Sustainability, applies to complex integrated systems comprising human components, e.g., society, economy, etc., and natural components such as ecosystems, climate, etc. Kates and Parris (2003) provide an insightful review on the changes and long-term trends in socioeconomic, environmental, and ecological systems and their relation to sustainability transition. These complex, diverse systems have a common characteristic, namely dynamic order, when looked at from an information point of view, in that they are orderly and wellorganized dynamic systems. Over time, they exist in a particular dynamic regime with a particular degree of dynamic order. But they are capable of shifting abruptly to different dynamic regimes with different degrees of dynamic order. Further, during the transition between two different regimes, there is typically a temporary loss of dynamic order. Afterwards, the system might shift to a different regime with either higher or lower dynamic order. Regime shifts can be defined as substantial, longterm reorganizations of complex systems such as societies, ecosystems, and climate (Carpenter and Brock 2006). Regime shifts have been documented and studied extensively in ecological systems (Steele 1998, Scheffer et al. 2001, Folke et al. 2004, Carpenter and Brock 2006, Mayer et al. 2006), social systems (Wood and Doan 2003, Kinzig et al. 2006), and climate systems (Miller et al. 1994). Quantifying and characterizing regime shifts is critical for understanding dynamics in ecosystems. Regime shifts, in the context of ecosystem resilience, could be a shift to an alternative basin of attraction by a stochastic event (Scheffer and Carpenter 2003). In ecology, some examples of regime shifts include eutrophication of lakes and coastal oceans, shifts among grassy and woodland

\footnotetext{
${ }^{1}$ U.S. Environmental Protection Agency, Office of Research and Development, National Risk Management Research Laboratory, Cincinnati, OH 45268, ${ }^{2}$ University of Arizona, Optical Sciences Center, Tucson, AZ 85721, ${ }^{3}$ RD Zande and Associates, Cincinnati, OH 45249
} 
cover types in rangelands, degradation of coral reefs, and regional climate change (Carpenter and Brock 2006). Quantification and classification of regime shifts is extremely difficult because they involve multiple causes; and hence, one must track multiple variables simultaneously over a long period of time. Not only is the study of regime shifts after its occurrence important but prediction of regime shifts through leading indicators is also critical. Recently there has been some significant work on the development of leading indicators for regime shifts (Kleinen et al. 2003, Carpenter and Brock 2006, van Nes and Scheffer 2007). Methods used for detection of dynamic regime shifts work very well on models, but the development of methods for real field data are lacking because of the large number of variables involved, noise in the field data, and sparseness of the datasets. There is a great need for indicators of regime shifts, particularly methods that are applicable to real ecosystems. In this context, it would be of immense value to develop methods having sound theoretical basis in order to detect and quantitatively characterize dynamic regimes and regime shifts.

Fisher information (Fisher 1922) is a fundamental quantity from which many known laws of nature can be derived (Frieden 2004). Our form of Fisher information has been used as a measure of dynamic order (Fath et al. 2003, Mayer et al. 2006). In this work we propose this latter form of the Fisher information along with a new robust calculation methodology as a measure of dynamic order in complex systems. The new method for calculating Fisher information from time series data is robust and applicable to sparse data sets with noise. We illustrate the principle with application to the detection and quantification of dynamic regime shifts in lake and marine ecosystems. Through Fisher Information, we also provide ways to classify dynamic regime shifts in terms of intensity and pervasiveness. Intensity is reflected by the degree of change in dynamic order, and pervasiveness is a reflection of how many observable variables of the system are affected by the change. These quantitative classifications of regime shifts are extremely important for ecosystem management. Furthermore, regime shifts often have large ecological and economical consequences, e.g., losses of water quality, declining of fish stocks, breakdowns of dryland agriculture (Carpenter and Brock 2006). Hence it is clear that, detecting and accessing the quality of dynamic regimes and regime shifts is very critical in identifying sustainable regimes. Hence, we provide a sustainable regime hypothesis that formally relates Fisher information, dynamic order, and regime shifts to sustainability. We believe that this work will improve our understanding of dynamic regimes and regime shifts in ecosystems and other complex systems and help establish Fisher information as a new and very powerful tool for sustainable environmental management and in policymaking.

\section{METHODS}

\section{Theory}

The statistician Ronald Fisher (Fisher 1922) developed a measure of indeterminacy, now called Fisher information. This is the information about the value of a parameter that is present in data $y$. Specifically, the Fisher information $I(\theta)$ is defined by,

$$
I(\theta) \equiv \int \frac{d y}{p(y \mid \theta)}\left[\frac{d p(y \mid \theta}{d \theta}\right]^{2}
$$

Here $p(y \mid \theta)$, often called the 'likelihood law,' is the probability density for obtaining a particular value of $y$ in the presence of $\theta$. Starting with Fisher's work, the Fisher information concept has been used as a unifying principle of physical laws (Frieden 2004). It also has been used as a measure of dynamic order in complex systems (Fath et al. 2003, Mayer et al. 2006).

When used as a measure of order in an environmental or ecological system, numerical values of the Fisher information $I(\theta)$ are required. However, Eq. 1, as it stands, is impractical for these purposes. This is because computing $I(\theta)$ requires numerical knowledge of the derivative $d p / d \theta$, and this depends upon the numerical value of the unknown parameter $\theta$. We develop below a means of addressing this issue as follows. Let the parameter $\theta$ be the ergodic mean $\theta=\langle y\rangle$ of $y$ given by 


$$
\langle y\rangle \equiv \frac{1}{T} \int_{0}^{T} y(t) d t
$$

where the integration is conducted over time $T$ included in all data observations. Thus, $I(\theta)$ is specifically the information about the ergodic mean of the data. Define a new variable $s=y$ - $\langle y\rangle$ representing fluctuations in $y$ from the mean. For the many cases common in environmental systems, it can be assumed to a reasonable approximation that within any particular dynamic regime the fluctuations $s$ are independent of the mean $\langle y\rangle$, i. e., the fluctuations about the mean are independent of the value of the mean. Note, however, that as a system approaches a transition between two different dynamic regimes, the fluctuations $(s)$ are likely to grow and become asymmetrical about the mean $\langle y\rangle$, and this would tend to make the fluctuations dependent on the mean. Hence, very near a regime transition, the aforementioned assumption would be an approximation. However, this has no implications for systems in stable dynamic regimes. Using elementary statistics (Frieden 2001), $p(y \mid\langle y\rangle)=p(y-\langle y\rangle \mid\langle y\rangle)=p(s \mid<$ $y>)=p(s)$ the latter by the definition of independence from the mean. Using these results and the chain rule of calculus gives

$$
\frac{d p}{d \theta}=\frac{d p}{d s} \frac{d s}{d \theta}=\frac{d p}{d s}
$$

And inserting in Eq. (1) gives directly

$$
I=\int \frac{d s}{p(s)}\left[\frac{d p(s)}{d s}\right]^{2}
$$

where the unknown parameter $\theta$ has now conveniently dropped out. The expression of Eq. 4 was previously derived by a slightly different route (Fath at al. 2003, Mayer et al. 2006). One further simplification often made is to substitute the probability density $p(s)$ with the amplitude $q(s)$ defined by $q^{2}(s) \equiv \mathrm{p}(\mathrm{s})$, to give

$$
I=4 \int d s\left[\frac{d q(s)}{d s}\right]^{2}
$$

This expression has the benefit of eliminating the division by $p(s)$, which can be numerically problematic if $p(s)$ happens to be a small number. The expression in Eq. 5 will be used in all our calculations.

Order in dynamic systems is conceptually associated with repeatability of observations. Hence, for a dynamic system that is perfectly orderly, repeated observations of the variables over time yield the same values within the limits of measurement uncertainty. Thus, for perfect order, the probability density function $(p(s))$ becomes a very sharp spike with a derivative $d p / d s$ that is approaching infinity, and a Fisher information (see Eq. 5) approaching infinity. This means that repeated measurements give increasingly more information eventually approaching infinite information. For a dynamic system that is perfectly disorderly, the opposite is true. Here all observations yield completely different and uncorrelated values for the variables, the likely hood of observing one value is the same as any other value, the probability density function $(p(s))$ is flat, the derivative $d p / d s$ is approaching zero, and the Fisher information is near zero. Real dynamic systems, however, exist between these two extremes of perfect order and perfect disorder and infinite and zero Fisher information. Hence, Fisher information is a theoretically sound measure of dynamic order.

Based on the previous arguments, we have expanded the Sustainable Regimes Hypothesis proposed by Cabezas and Fath (2002) for use in environmental management. The hypothesis in summary states that: (1) dynamic systems in a sustainable regime show a time-averaged Fisher information that does not change with time; (2) steadily declining Fisher information in which a system is progressively shifting to successive regimes, each with lower Fisher information, signifies a loss of dynamic order and organization; (3) a steadily rising Fisher information in which a system is shifting to successive regimes, each with higher Fisher information, simply indicates increasing dynamic order; and (4) the interval or shift between two dynamic regimes is characterized by a steep drop in dynamic order and Fisher information. However, in this context, sustainable regimes should not be construed as being necessarily agreeable with human preferences. This 
paper essentially deals with the fourth point of the sustainable hypothesis with which Fisher information is used to detect and quantify dynamic regime shifts in ecological systems.

\section{Methodology}

Previously Fisher information has been calculated from Eq. 5 using a methodology that requires the evaluation of the first and second time derivatives of the time series (Fath et al. 2003, Cabezas et al. 2005, Mayer et al. 2006). The main problem here was obtaining accurate second derivatives from the noisy and sparse data sets often found in real systems. This made it extremely difficult to separate natural fluctuations from noise, and the detection of regime shifts proved difficult. The exception was when the analysis involved food web models with many model generated data points and no noise, or when the Fisher information calculation for a real system was averaged over a large block of time to smooth out errors. This averaging made it possible to demonstrate that Fisher information changes with regime shifts in real systems, but locating the time at which the regime shift occurred was not possible. Our present effort aims to develop a robust methodology that can be used to characterize and detect regime shifts, i.e., the time at which the shift happened, using field data from real, complex systems with sparse data sets and noise. Locating the time of regime shifts is critical for using the methodology in managing real systems.

The critical steps in this new methodology for computing the Fisher information are: (1) dividing the time series into a sequence of time windows, (2) within each time window binning the data points into states according to the methodology below, (3) constructing a probability density function based on the likelihood of observing the system states within each time window, and (4) calculating Fisher information from the probability-density function using Eq. 5 for each time window.

We expand on some of these critical steps within each time window in the following paragraphs. First, to define the system, one has to select variables, i.e., state variables that characterize the state of the system. For a system represented by $n$ state variables $x_{i}$, the system can be said to have a trajectory in a phase space of n-dimensions and time. At any particular time, the values of all the variables define a particular point in the system trajectory in phase space. Because all measurements have some uncertainty, the values of $x_{i}$ are known only within some uncertainty $\Delta x_{i}$. Hence, any two values $\left(x_{i}\left(t_{\mathrm{j}}\right)\right.$ and $\left.\mathrm{x}_{i}\left(t_{j}\right)\right)$ for the same variable $x_{i}$ at two different times $t_{i}$ and $t_{j}$ are indistinguishable from each other if $\left|x_{i}\left(t_{\mathrm{i}}\right)-\mathrm{x}_{i}\left(t_{j}\right)\right| \leq \Delta x_{i}$. If any two values are indistinguishable, they can be interpreted as two observations of the same value of $x_{i}$. Second, the system trajectory in phase space is divided into a sequence of time windows, and the trajectory within each time window is discritized into a number of different states. These states are essentially ndimensional hyper rectangles centered on particular data points as further elaborated below. Here the lengths of the ' $n$ ' sides of the hyper rectangles are determined by the uncertainties ' $\Delta \mathrm{x}_{i}$ ' in the state variables. These hyper rectangles are states of the system. For cases in which the measurement uncertainty $\Delta \mathrm{x}_{i}$ is unknown or unreported, we estimate the uncertainty as follows. The size of a state, i.e., lengths of the hyper rectangles sides, is determined from a small data region in the time series where we know that the system has been in a stable dynamic regime. Here we assume that any variations over this period can be attributed to uncertainty, $\Delta \mathrm{x}_{i}$ 's and/or natural cyclical variations, but not to a dynamic regime change. We then compute the standard deviation $\left(\sigma_{i}\right)$ for each state variable $x_{i}$ from that small patch of the data, and then we set the size of the sides of the state hyper rectangle by $\Delta \mathrm{x}_{i}= \pm 2 \sigma_{i}$. According to Chebyshev's theorem this will encompass at least $89 \%$ of the data points regardless of the probability distribution form. Chebyshev's theorem (Lapin 1975) formally states that "The proportion of observations falling within $k$ standard deviations of the mean is at least $1-1 / k^{2}$." Third, within the first time window, we next distribute or "bin" each temporal data point in the system trajectory into different states, i.e., hyper rectangles. Note that each data point is an $n$ dimensional vector whose coordinates are $\left(x_{1}(\mathrm{t}), \mathrm{x}_{2}\right.$ $\left.(\mathrm{t}), \ldots \mathrm{x}_{i}(t)\right)$ at a time $(t)$. Then, the first point in time in the system trajectory is taken as the center of the first state and a state hyper rectangle is constructed around it. All the points falling within the boundaries of the first state are counted or binned together. Fourth, the next uncounted point within the time window is selected, the second state is built around it, and the points are binned as before. Finally, this procedure is repeated until all the points are binned into states within the current time window, and the process moves to the next time window. 
The aforementioned procedure of binning would work ideally in the case of a system in which the state variables are well known and truly independent, and when the measurement uncertainty associated with each variable is well known. Unfortunately, these conditions are not often met for the kinds of real systems, e.g., ecosystems, economies, etc., of interest. Rather, a large number of suitable variables, say 100, are usually selected to characterize the system knowing that some may be correlated or redundant and with unknown uncertainty. This results in states with a large number of dimensions (100) defined by hyper rectangles of questionable boundaries. In such a situation, when attempting to determine whether two points, $\left[x_{1}\left(t_{1}\right), x_{2}\left(t_{1}\right), \ldots x_{100}\left(t_{1}\right)\right]$ and $\left[x_{1}\left(t_{2}\right), x_{2}\left(t_{2}\right)\right.$ ,... $\left.x_{100}\left(t_{2}\right)\right]$, are to be binned together in a state, there often are a few coordinates, say five variables, whose values do not fall within the boundary of the hyper rectangle. However, the values of the remaining state variables, say 95 variables, fall within the given state. Given the uncertainty associated with the variables and the determination of the $\Delta \mathrm{x}_{i}$ 's and consequently the state boundaries, it would seem reasonable to bin points together if 95 out of 100 variables so indicated. To address this issue, we introduced a flexible tightening of the binning requirements. This specifies a percentage, say $80 \%$, of total state variables that need to have their values within their respective state boundaries for the point to be classified in that particular state. We call this term tightening level.

The tightening process is as follows, for a given tightening level, after binning all the points into states, the states are rearranged according to their closeness in phase space, and then the number of points in each state is counted resulting in a probability distribution function for the states. Since there is no natural way of setting the tightening level, we bin at different tightening levels, starting from a very relaxed condition, say $20 \%$, to a very strict condition, say $90 \%$. For a very relaxed tightening, all data points fall in the same state, and for very strict tightening all data points fall in different states. In practice, rather than set the lower bound arbitrarily, we select the lowest tightening level at which we notice more than one state present, and fix a suitable upper limit. We then do multiple calculations, one for each tightening level, between these two extremes. This results in multiple probability density functions $p(s)$ and, from Eq. 5, multiple Fisher information values, one for each tightening level. Finally, we average all of the Fisher information values within a given time window, and report this arithmetic mean as our estimate of the Fisher information for that time window. Moreover, by varying the tightening level we can quantify and analyze the pervasiveness of the regime shift as demonstrated in the application results.

To detect regime shifts, we compare the average Fisher information from one time window to the next. More specifically, we compute the Fisher information over a specified time window $\left((t){ }_{1}\right.$ to $(t)$ ${ }_{10}$ ), move the time window by a specified number of time steps, such that there is an overlap, and then we compute the average Fisher information over the new time bracket $\left((\mathrm{t})_{5}\right.$ to $\left.(\mathrm{t})_{15}\right)$. This is important because, according to the Sustainable Regimes Hypothesis, there is no regime change if there is no change in the value of the time averaged Fisher information. If there is a drop in Fisher information, there has likely been a regime change.

\section{RESULTS}

\section{Model System: shallow lakes}

To illustrate the application of Fisher information in detecting dynamic regime shifts, we selected a simple model system, shallow lakes that shift between eutrophic and oligotrophic regimes due to inflow of phosphorus. One reason for the choice is that the system can be characterized by one variable, and we can plot the system dynamics and visually demonstrate the regime change. These types of systems have been studied (Carpenter 2003, Carpenter et al. 1999), and some seminal work on the application of these statistics to regime change has been done (Pawlowski and Cabezas 2008).

The ecosystem in lakes depends largely on the available nutrients with phosphorous often being the limiting nutrient. In lakes, phosphorous occurs through natural means as well as through human contribution in the form of sewage and runoff from fertilizer used in agriculture (Maler 2000). The flow of phosphorous results in the growth of algae that can change a clear lake with a diversity of fishes to a turbid lake full of algae with general reduction in fish stocks (Maler 2000). The clear lake is in an oligotrophic regime whereas the turbid lake is in a eutrophic regime. Needless to say humans often would want to sustain oligotrophic regimes. Remarkably, water clarity often seems to be hardly affected by increased nutrient concentration until a 
critical threshold is passed, at which point the lake shifts abruptly from clear to turbid (Scheffer et al. 2001). The dynamics of the eutrophication process of shallow lakes was modeled from the work of Carpenter (2003) and Maler (2000) (also used by Pawlowski and Cabezas 2008). The model gives,

$$
\begin{gathered}
x_{t+1}=a e^{z_{t}}-b x_{t}+\frac{x_{t}^{2}}{1+x_{t}^{2}} \\
Z_{t+1}=p Z_{t}+\varepsilon_{t}
\end{gathered}
$$

Where $\mathrm{X}$ is the stock of phosphorous in algae in the lake at time $t$, ' $a$ ' is the input of phosphorous into the lake, $b$ is a constant describing the natural removal of phosphorous from the water, and the exponential term serves to add noise. Here $p$ is a constant, and $\varepsilon_{\mathrm{t}}$ is normally distributed (red) noise with a mean of zero and a variance of 0.0002 . Red noise is typically a random noise of low frequency. We carried out simulations of this model with $b=0.58, p=0.99$ and the following parameter changes,

$$
\begin{array}{ll}
0<t<200 & a=0.02 \\
200<t<210 & a=0.02+\left(\frac{t-200}{10}\right) * 0.06 \\
210<t<500 & a=0.02+0.06=0.08 \\
500<t<1800 & a=0.08+\left(\frac{t-500}{1300}\right) * 0.06 \\
1800<t & a=0.08+0.06=0.14
\end{array}
$$

Three different scenarios were generated in order to test and demonstrate the proposed methodology in relation to the difficulties of real time series data sets. The first corresponds to the ideal case where there is plenty of data and no noise, which is modeled by setting $Z=0$. The lake dynamics and the Fisher information plot, almost exactly the same as Fig. 1b, are not shown here for brevity. The second scenario corresponds to the situation in which there is a lot of data but there is significant noise in the data ( $Z$ from Eq. 7). The resultant dynamics is shown in Fig. 1a. In lakes, variance due to fluctuations in recycling is difficult to discriminate from variance due to noisy nutrient inputs (Carpenter and Brock 2006). The third scenario corresponds to a data set that is both sparse and has significant noise ( $Z$ from
Eq. 7). This is commonly encountered with field data. The lake dynamics were simulated by taking a time step of 50, instead of 1 , and adding noise as already discussed. This is shown in Fig. 2a. The size of the state was determined using the first 500 time points in the case of the first and second scenarios, whereas the first 10 points were used in the case of third scenario. The Fisher information was integrated over a time window of 100 steps, and the time window was moved by 50 steps, resulting in an overlap of 50 time steps. Since this system is represented by only one variable, the tightening level in the Fisher information calculation becomes redundant and is not used. The time averaged Fisher information from model generated data for the second and third scenarios was calculated using the methodology described above, and the corresponding Fisher information plots are shown in Figs 1b, and $2 \mathrm{~b}$, respectively.

\section{Real system: Bering Strait marine ecosystem}

The biological response to climate variability in the atmosphere and ocean are largely unknown. However, it is widely accepted that marine populations respond to climatic changes (McGowan et al. 1998, Hsieh et al. 2005, Harley et al. 2006). The interdecadal changes in marine atmospheric conditions, characterized by the Pacific Decadal Oscillation (PDO), are a pan-Pacific phenomenon associated with climate changes in the Pacific. The PDO involves sudden, abrupt transitions in atmospheric-marine physical conditions that are stable and lasts for 20-35 yr (Hilborn et al. 2003). Marine ecosystems seem to respond to changes that occur under marine physical conditions associated with the PDO. Many biological features of the North Pacific Ocean show significant changes between interdecadal phases of a PDO. The prolonged changes in ecosystem organization associated with changes in atmospheric-oceanic coupling are thought to constitute regime changes (Hilborn et al. 2003). It is widely believed that a regime shift occurred in the North Pacific Ocean and Bering Sea in the mid 1970's, which had widespread consequences for the biota (Hare and Mantua 2000). Several studies have documented evidence on the climatic (Graham 1994, Miller et al. 1994), as well as the resultant biological changes (Francis and Hare 1994, McGowan et al. 1998, Francis et al. 1998, McGowan et al. 2003, Grebmeier et al. 2006). The marine science community lacks widely 
Fig. 1. a) Model solution for lake phosphorous concentration in the case of red noise b) Time averaged Fisher Information.
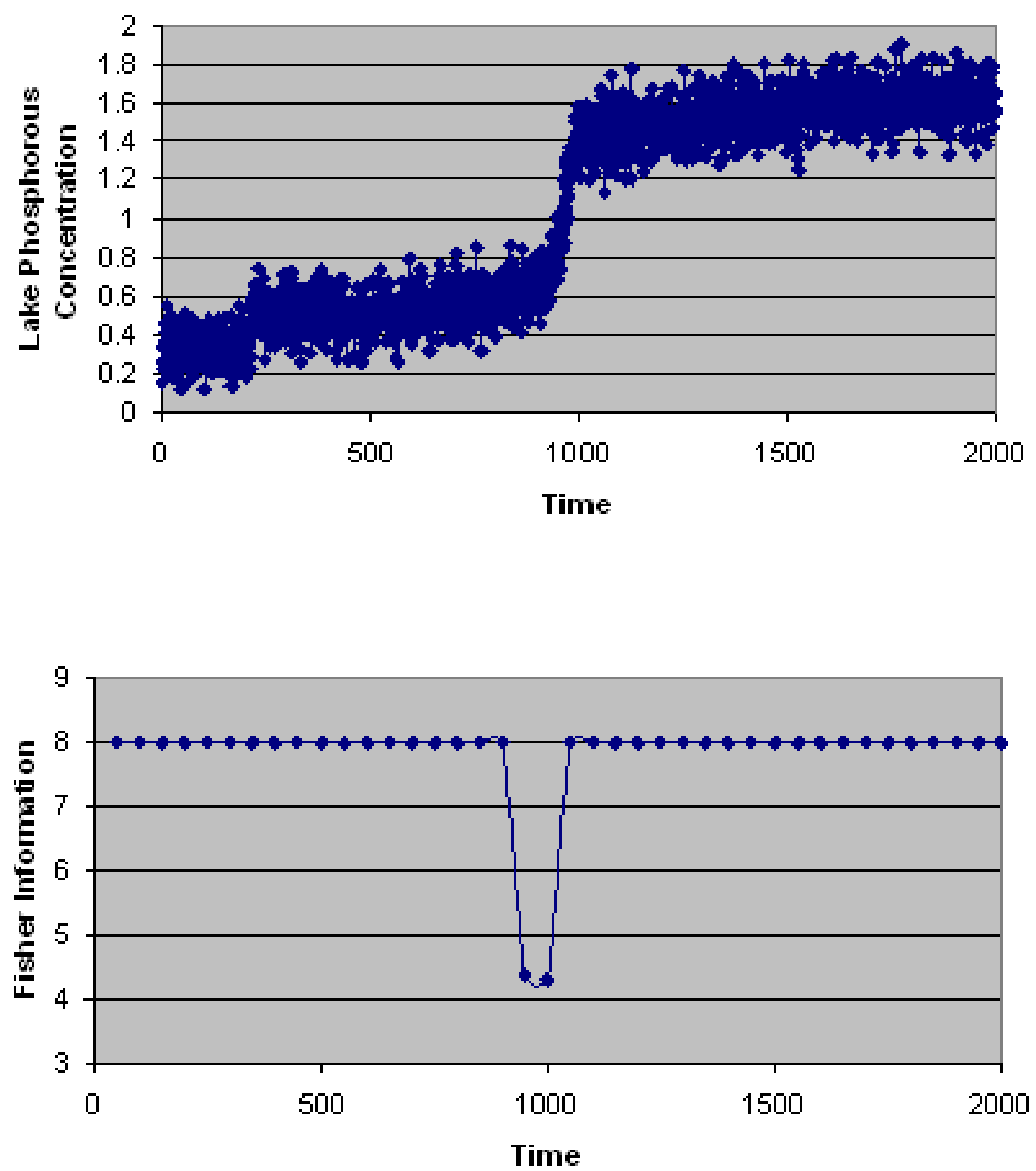
Fig. 2. a) Model solution for lake phosphorous concentration in the case of sparse data and red noise b) Time averaged Fisher information.
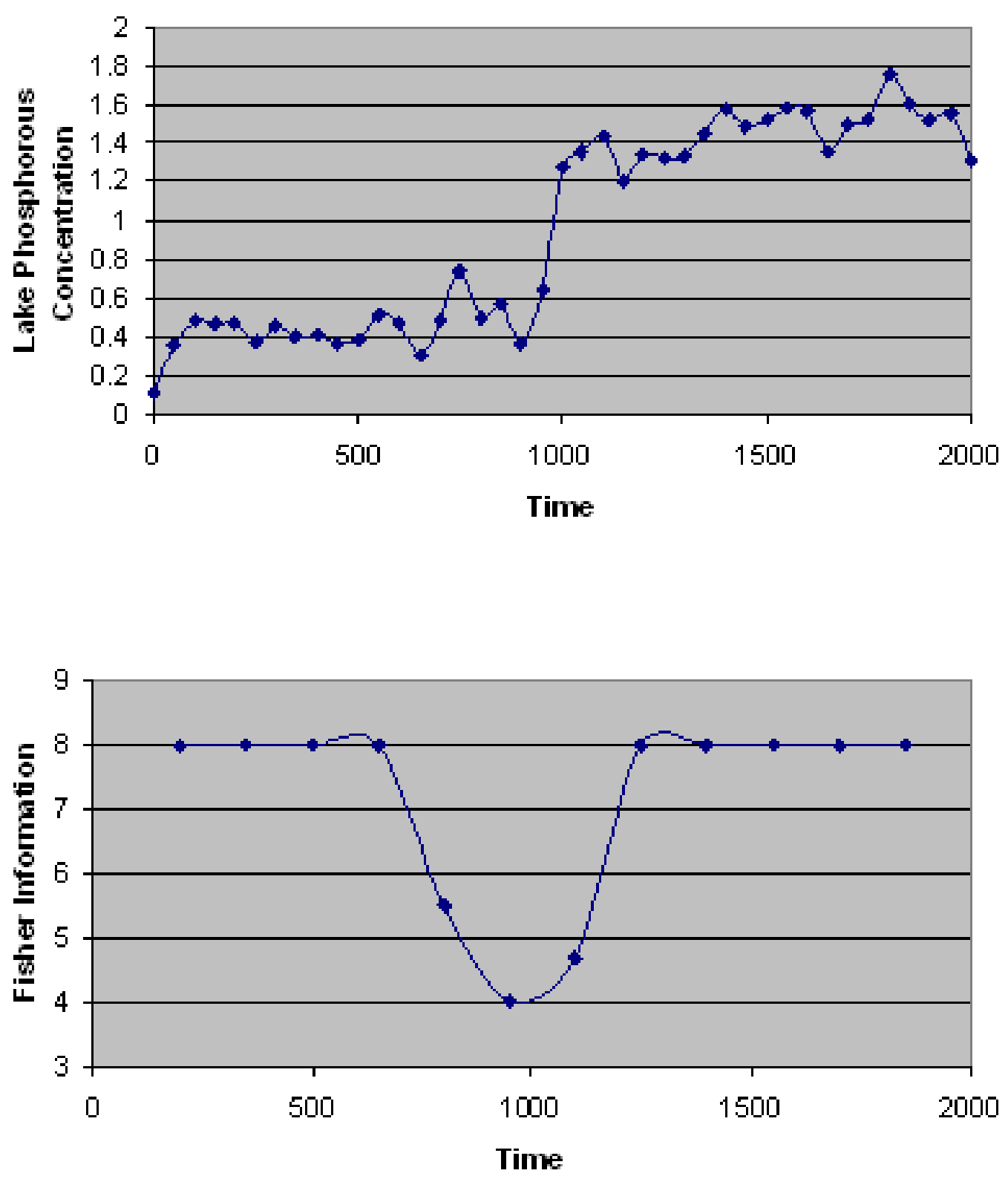
Fig. 3. Fisher information: climate system.

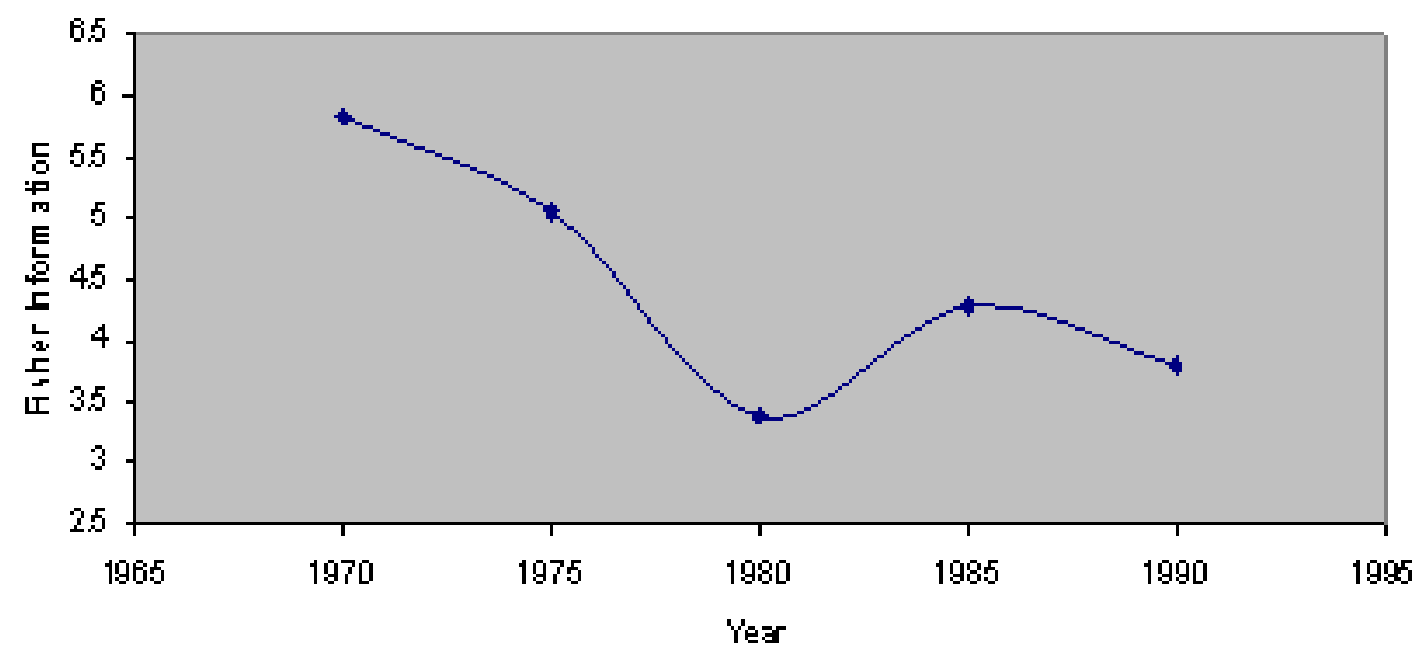

accepted methods and tools for detecting regime shifts in marine ecosystem data sets (Mantua 2004). Note that Mantua (2004) has reviewed various methods for detecting regime shifts in marine ecosystems. Evidence of climate shifts can be collected from air temperatures, sea surface temperatures, PDO, and ENSO indices, ice cover, etc. Biological responses seem to be related to changes in marine phytoplankton productivity that are transmitted through zooplankton to fishes (Hilborn et al. 2003). These biological responses seem to manifest in zooplankton biomass, sockeye salmon populations, shrimp populations, etc. Hare and Mantua (2000) assembled data with 100 environmental time-series variables data from 1965-1997, which included 31 climatic variables and 69 biological variables. Through the analysis of this data, they have identified a further regime shift in 1989 in some components of the North Pacific Ocean.

We used Fisher information analysis based on the methodology presented in this work on the Hare and Mantua (2000) data set to detect and analyze the climate, biological, and coupled climate-biological regime shifts in the North Pacific Ocean and the Bearing Strait. Our previous work (Mayer et al. 2006) showed that a difference in the Fisher information exists between regimes, but it required that the point in time when the regime change occurred be known a priori. Here we are able to use the new methodology to estimate the approximate location in time of the regime change from the drop in Fisher information. For our analysis, we used 65 variables that had complete time series data. Among these, 30 were climate variables and 35 were biological variables. The time period used in the analysis was from 1965 to 1995 . To bin the time series data into states in phase space, the size of the states for each dimension, i.e., each variable, was determined using the first five points in the time series, i.e., from 1965 to 1969 , since it is broadly considered that the system was in a stable regime during this period. The size of the states was fixed at $\pm 2 \mathrm{SD}$ calculated from these five points. The Fisher information was integrated over a time window of $10 \mathrm{yr}$, and the time window was moved in 5-yr steps, resulting in an overlap of $5 \mathrm{yr}$ in each calculation. The time-dependent Fisher information was calculated for four different tightening levels namely $50 \%, 60 \%, 70 \%$, and $80 \%$. The final timedependent Fisher information was calculated as an arithmetic mean of these four levels. Figure 3 shows the time-averaged Fisher information values for the climate variables. Figure 4 shows the time-averaged Fisher information values for the biological 
Fig. 4. Fisher information: biological system.

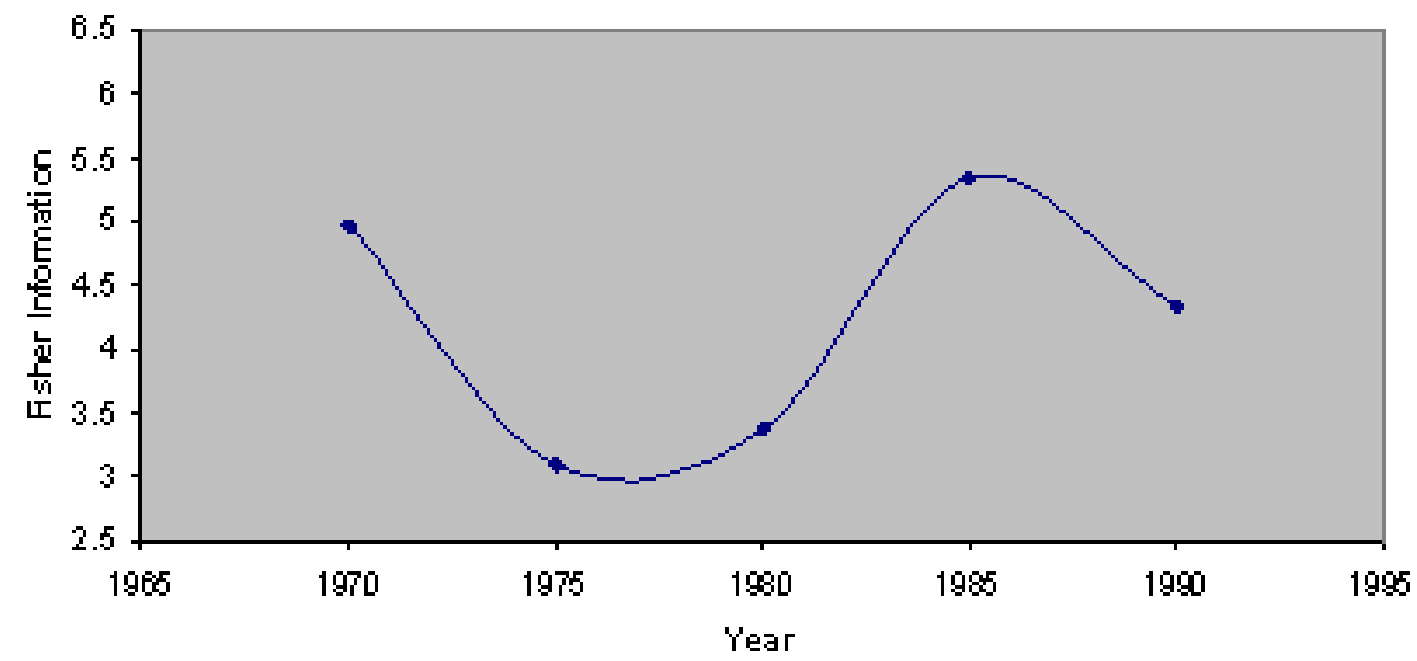

variables. From Figs 3 and 4, we can clearly see that in both the climatic and biological components there is a significant drop in Fisher information, whose minimum appears close to 1977 . Then there is a gain in Fisher information suggesting that there was a return to a stable regime after 1977, and then the Fisher information drops corresponding to the 1989 regime shift. Here it is important to note that for each time-averaged Fisher information calculation, we have used 10 data points with an overlap of 5 points, so it is not possible to estimate the exact year in which the regime shift occurred. But we could predict a time window in which the minimum Fisher information lies and say that the regime shift occurred within that time bracket. For the end points, the first Fisher information value corresponding to the year 1970 and the final Fisher information value corresponding to the year 1990, in Figs 3, 4 and 5 were calculated using time series data from 1965 to 1975 and 1985 to 1995 , respectively. Next we carried out a Fisher information calculation using all 65 variables together to study the regime shifts in the coupled system. Figure 5 shows the plot of this timedependent Fisher information calculation. Here, similar to the climatic and biological regime shifts, we could detect the 1977 and 1989 regime shifts. Finally, Figs 6 (a-d) show different Fisher information plots corresponding to $40 \%, 50 \%, 60 \%$, and $100 \%$ tightening levels.

\section{DISCUSSION}

The ability to use Fisher information as a means of detecting and assessing dynamic regime changes depends on the ability of the methodology to provide reliable Fisher information values from real time series data sets on complex systems. More often than not, these data sets contain significant noise and measurement uncertainties that are either not reported or not quantifiable. Moreover, temporally these datasets are invariably sparse in nature. However, the approach of building the states based on measurement uncertainties, quantified by the standard deviation and the application of Chebyshev's theorem, and the use of the tightening level methodology for binning the points in state space inherently addresses the issue of measurement uncertainty. The ability of this methodology to handle noisy and sparse data sets was demonstrated with the help of the Lake Model system in section 3.1. Figure 1 shows that the methodology was able to use noisy data sets while producing useful results, and Fig. 2 shows the ability of the methodology to 
Fig. 5. Fisher information: coupled climate, biological system.

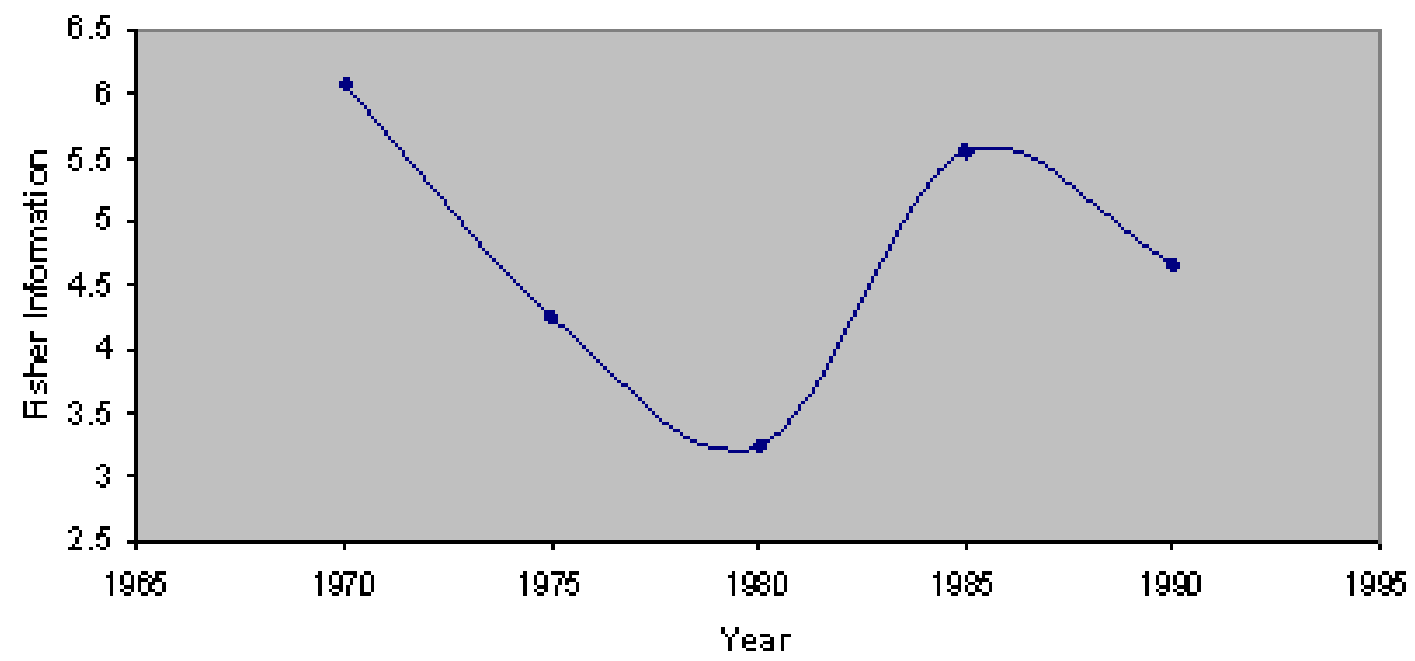

manage sparse-noisy data sets as well. In both of the cases, there was a significant drop in the Fisher information corresponding to the regime shift from oligotrophic to eutrophic condition as expected. However, in the case of sparse-noisy dataset the drop in Fisher information was over a broader time horizon (Fig. 2b) as expected, but nevertheless the regime shift was clearly detected. Note that the Fisher information was the same for both oligotrophic and eutrophic lake conditions, signifying that both of these regimes had similar dynamic order. This is due to the use of a simple one-dimensional model for the lake regime as against a more complex model with many dimensions. However, two different regimes generally have different dynamic order and different Fisher information, separated in time by a steep drop in Fisher information during the transition phase.

Since a critical issue in sustainability is that of maintaining a regime of the Earth that is amenable to supporting human life, three critical subissues arise: (1) detecting regime shifts, (2) determining the intensity of the regime shift, and (3) estimating the pervasiveness of the regime shift. The occurrence of a regime shift can be detected conclusively if there is a significant drop in dynamic order, as measured by Fisher Information. The intensity of the regime shift can be determined by the level of the drop in Fisher information, i.e., an intense regime shift will have a steeper drop in Fisher information value as against a milder regime shift. The pervasiveness of the regime shift, characterized by shifts across a wide number of state variables, can be determined by changing the tightening level, i.e., a more pervasive shift is manifested across more variables. That is, a significant drop in Fisher information at a lower tightening level will indicate a highly pervasive regime shift, and a regime shift that is only manifested at higher tightening levels is likely to be less pervasive.

These can be clearly demonstrated through Figs 3, 4 , and 5, in the case of the climate-biological regime shifts in the Bering Strait. Here we can clearly see a drop in Fisher information corresponding to the 1977 and 1989 regime shifts. From these figures, we can also see that the drop in Fisher information corresponding to the 1977 regime shift is larger in magnitude as compared to the 1989 regime shift. This leads us to believe that the 1977 shift was more intense than the 1989 shift. When we compare the physical system (Fig. 3) and the biological system (Fig. 4), we can see that the shifts are reflected more clearly and consistently by the biological data than 
Fig. 6. Fisher information: different tightening levels (TL) a) TL: 40\%, b) TL: 50\% c), TL: 60\%, d) TL: $100 \%$.
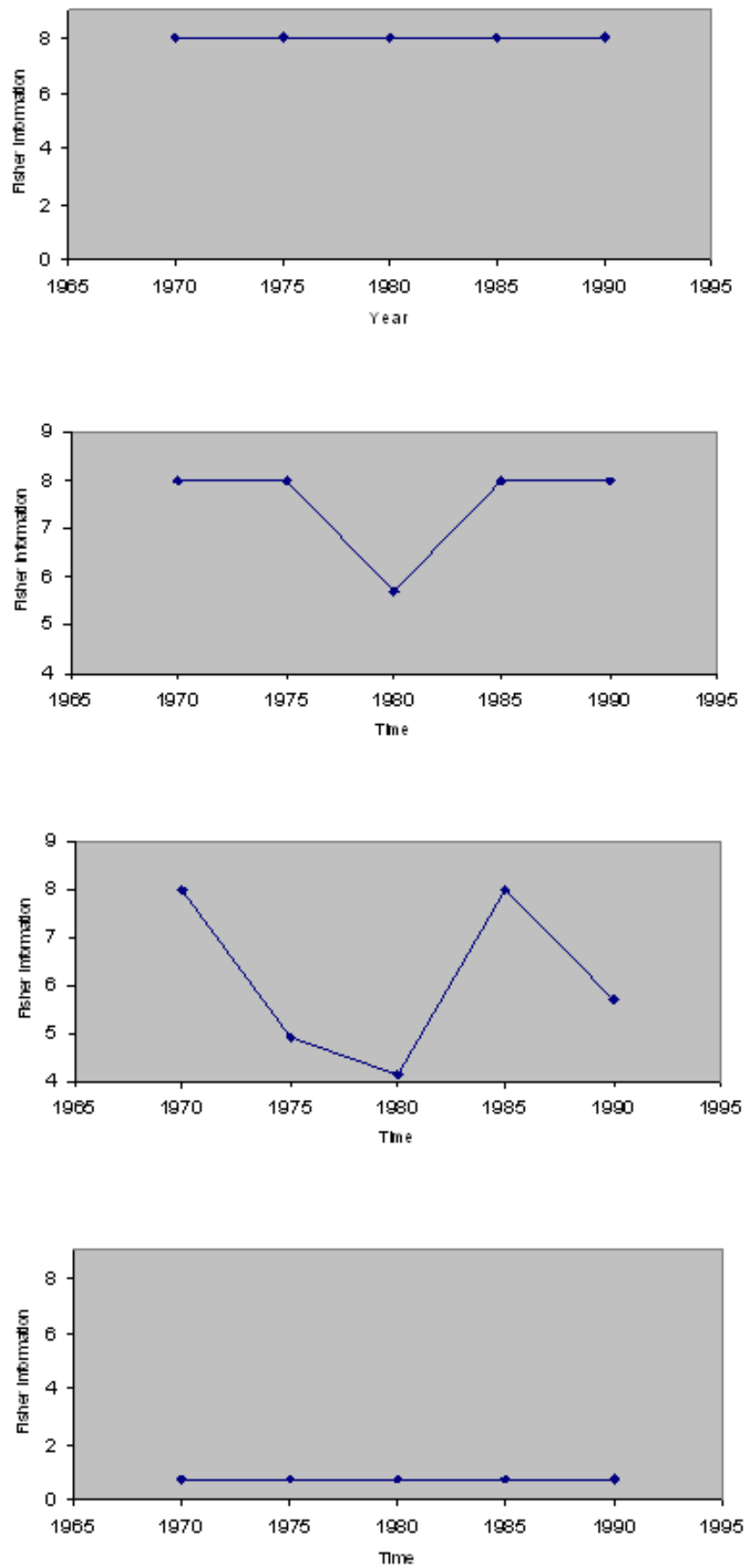
by the physical data. As discussed by Scheffer et al. (2001) this could suggest that biotic feedbacks might be stabilizing the community in a certain regime and that shifts to a different regime are triggered merely by physical forcing. From Fig. 6a we can see that at a low tightening level of $40 \%$, $i$. e., a very relaxed condition, all the points are binned together as expected in a single state, and we have maximum and constant Fisher information over the entire time period. At this tightening level we could not detect either of the regime shifts. When we increase the tightening level to $50 \%$, we can see a drop in Fisher information corresponding to the 1977 regime shift but not the 1989 regime shift (Fig. $6 \mathrm{~b})$. Note that a $50 \%$ tightening level is still a relatively relaxed condition, and even at this relaxed condition we can detect the 1977 regime shift but not the 1989 shift. This indicates that the 1977 regime shift was more pervasive compared to the 1989 shift. When we increase the tightening level to $60 \%$, we can see a drop in Fisher information, corresponding to both the 1977 and 1989 regime shifts (Fig. 6c). As expected for a tightening level of $100 \%$, all the points were binned into separate states (Fig. 6d), and very low Fisher information was observed for the entire time period. Neither of the regimes shifts could be detected at this level. This shows the necessity of using different tightening levels to account for the tremendous uncertainty when working with real systems.

\section{CONCLUSION}

In summary, the chief goal of sustainable environmental management is to promote dynamic regimes that are favorable to human existence. A fundamental property common to all of these systems is dynamic order, e.g., well-organized systems have a high degree of dynamic order, and different regimes of the same system have different degrees of dynamic order. Fisher information captures dynamic order in these systems. When a system experiences a regime shift, it is typically manifested as a drop in Fisher information. Thus, Fisher information can be used as a measure of dynamic order and as a means of detecting regime shifts. Further, Fisher information can be used for determining, (1) the intensity of the regime shifts, and (2) the pervasiveness of the regime shift. Hence, we propose the methodology as a practical and quantitative means of classifying dynamic regime changes. This was shown from the Fisher information calculations for the Bering Sea, which seems to respond to climate-ocean variability. We were able to detect two well-documented regime shifts that are thought to have occurred in 1977 and 1989. From the analysis we believe that the 1977 shift was more intensive and pervasive as compared to the 1989 shift. Even though this paper essentially addresses the detection and quantification of regime shifts that have already occurred, Fisher Information holds promise as a leading indicator that could provide warning of regime shifts so that proper ecosystem management decisions can be taken.

Responses to this article can be read online at:

http://www.ecologyandsociety.org/voll3/iss 1/art22/responses/

\section{Acknowledgments:}

The authors are grateful to $S$. R. Hare and N. J. Mantua for providing data from their study of the North Pacific Ocean. The authors also acknowledge the seminal work of A. L. Mayer and B. D. Fath, their former colleagues at the U.S. EPA, on the application of information theory to the ecological regime changes.

\section{LITERATURE CITED}

Cabezas, H., and B. D. Fath. 2002. Towards a theory of sustainable systems. Fluid Phase Equilibria 2:194-197.

Cabezas, H., C. W. Pawlowski, A. L. Mayer, and N. T. Hoagland. 2005. Sustainable systems theory: ecological and other aspects. Journal of Cleaner Production 13:455-467.

Carpenter, S. R. 2003. Regime shifts in lake ecosystems: pattern and variation. Volume 15 , Excellence in Ecology Series, Ecology Institute, Oldendorf/Luhe, Germany.

Carpenter, S. R., and W. A. Brock. 2006. Rising variance: a leading indicator of ecological transition. Ecology Letters 9:311-318.

Carpenter, S. R., D. Ludwig, and W. A. Brock. 1999. Management of eutrophication for lakes subject to potentially irreversible change. 
Ecological Applications 9:751-771.

Fath, B. D., H. Cabezas, and C. W. Pawlowski. 2003. Regime changes in ecological systems: an information theory approach. Journal of Theoretical Biology 222:517-530.

Fisher, R. A. 1922. On the mathematical foundations of theoretical statistics. Philosophical Transactions of the Royal Society of London 222:309-368.

Folke, C., S. Carpenter, B. Walker, M. Scheffer, T. Elmqvist, L. Gunderson, and C. S. Holling. 2004. Regime shifts, resilience, and biodiversity in ecosystem management. Annual Review of Ecology Evolution and Systematics 35:557-581.

Francis, R. C., and S. R. Hare. 1994. Decadalscale regime shifts in the large marine ecosystems of the north-east Pacific: a case for historical science. Fisheries Oceanography 3:279-291.

Francis, R. C., S. R. Hare, A. B. Hollowed, and W.S. Wooster. 1998. Effects of interdecadal climate variability on the oceanic ecosystems of the NE Pacific. Fisheries Oceanography 7:1-21.

Frieden, B. R. 2001. Probability, statistical optics and data testing. Springer-Verlag, New York, New York, USA.

Frieden, B. R. 2004. Science from Fisher information: a unification. Cambridge University Press, Cambridge, UK.

Graham, N. E. 1994. Decadal-scale climate variability in the tropical and North Pacific during the 1970s and 1980s: observations and model results. Climate Dynamics 10:135-162.

Grebmeier, J. M., J. E. Overland, S. E. Moore, E. V. Farley, E. C. Carmack, L. W. Cooper, K. E. Frey, J. H. Helle, F. A. McLaughlin, and S. Y. McNutt. 2006. A major ecosystem shift in the northern bearing sea. Science 311:1461-1464.

Hare, S. R. and N. J. Mantua. 2000. Empirical evidence for north pacific regime shifts in 1977 and 1989. Progress in Oceanography 47:103-145.

Harley, C. D. G., A. R. Hughes, K. M. Hultgren, B. G. Miner, C. J. B. Sorte, S. Thornber, L. F. Rodriguez, L. Tomanek, and S. L. Williams.
2006. The impacts of climate change in coastal marine ecosystems. Ecology Letters 9:228-241.

Hilborn, R., T. P. Quinn, D. E. Schindler, and D. E. Rogers. 2003. Biocomplexity and fisheries sustainability. Proceedings of National Academy of Sciences USA. 100:6564-6568.

Hsieh, C., S. M. Glaser, A. J. Lucas, and G. Sugihara. 2005. Distinguishing random environmental fluctuations from ecological catastrophes for the North Pacific ocean. Nature 435:336-340.

Kates, R.W., and T. M. Parris. 2003. Long term trends and a sustainability transition. Proceedings of National Academy of Sciences USA. 100:8062-8067.

Kinzig, A. P., P. Ryan, M. Etienne, H. Allison, T. Elmq vist, and B. H. Walker. 2006. Resilience and regime shifts: assessing cascading effects. Ecology and Society 11 (1). [Online] URL: http://www.ecol ogyandsociety.org/vol11/iss1/art20/.

Kleinen, T., H. Held, and G. Petschel-Held. 2003. The potential role of spectral properties in detecting thresholds in the earth system: application to the thermohaline circulation. Ocean Dynamics 53:53-63.

Lapin, L. L. 1975. Statistics: meaning and method. Harcourt Brace Jovanovich, New York, New York, USA.

Maler, K. G. 2000. Development ecological resources and their management: a study of complex dynamic systems. European Economic Review 44:645-665.

Mantua, N. 2004. Methods for detecting regime shifts in large marine ecosystems: a review with approaches applied to North Pacific data. Progress in Oceanography 60:165-182.

Mayer, A. L., C. W. Pawlowski, and H. Cabezas, H. 2006. Fisher information and dynamic regime changes in ecological systems. Ecological Modelling 195:72-82.

McGowan, J. A., S. J. Bograd, R. J. Lynn, and A. J. Miller. 2003. The biological response to the 1977 regime shift in the California current. Deep-Sea Res II 50:2567-2582.

McGowan, J.A., D. R. Cayan, and L. M.Dorman. 
1998. Climate-ocean variability and ecosystem response in the North Pacific ocean. Science 281:210-217.

Miller, A. J., D. R. Cayan, T. P. Barnett, N. E. Graham, and J. M. Oberhuber. 1994. The 1976-77 climate shift of the Pacific Ocean. Oceanography 7:21-26.

Pawlowski, C. W., and H. Cabezas. 2008. Identification of regime shifts in time series using neighborhood statistic. 5:30-36.

Scheffer, M., and S. R. Carpenter. 2003. Catastrophic regime shifts in ecosystems: linking theory to observation. Trends in Ecology and Evolution 18:648-656.

Scheffer, M., S. Carpenter, J. A. Foley, C. Folke, and B. H. Walke. 2001. Catastrophic shifts in ecosystems. Nature 413:591-596.

Steele, J. H. 1998. Regime shifts in marine ecosystems. Ecological Applications 8:S33-S36.

van Nes, E. H., and M. Scheffer. 2007. Slow recovery from perturbations as a generic indicator of a nearby catastrophic shift. The American Naturalist 169:738-747.

Wood, B. D., and A. Doan. 2003. The politics of problem definition: applying and testing threshold models. American Journal of Political Science 47:640-653. 